\title{
Long-Term Performance Evaluation of Main Cable for Large Span Suspension Bridge Based on ARIMA Model
}

\author{
Shiqiao XU ${ }^{\mathrm{a}, 1}$, and Rujin $\mathrm{MA}^{\mathrm{b}}$ and Chuanjie CUI ${ }^{\mathrm{b}}$ \\ ${ }^{a}$ China Construction Eighth Engineering Division Corp., Ltd, Shanghai, China \\ ${ }^{\mathrm{b}}$ Department of Bridge Engineering, Tongji University, Shanghai, China
}

\begin{abstract}
Main cables are major load-bearing components and play a decisive role in the long-term performance of suspension bridges. In particular, the wide application of health monitoring systems in large-span bridges facilitates a new channel for the performance evaluation of bridges. Under this context, Xihoumen Bridge is taken as the engineering research background. The long-term performance of the bridge's main cable was evaluated by analyzing the main cable strand force data from January 2015 to October 2020, which was collected by the anchor rope meter. Firstly, the difference calculation was performed on the basis of the modeling theory of stationary time series to realize the stationary process of the original time series. Then, the Autoregressive Integrated Moving Average (ARIMA) model is established to predict the main cable strand force of the Xihoumen Bridge by model order estimation and parameter identification. Finally, the load degree index is defined in the discussion part to evaluate the long-term performance and determine the performance grading of the main cable.
\end{abstract}

Keywords. Large span suspension bridge, long-term performance evaluation, main cable strand force, arima model, monitoring data

\section{Introduction}

Three factors determine and characterize the reliability of a bridge throughout its life cycle: safety performance, service performance, and durability performance. Main cable is one of the most important force bearing and transmission components of a suspension bridge that is always under the state of high stress [1]. Furthermore, the main cables are arranged outside the beam body and they are sensitive to the external environment, which increases the main cable's proneness to diseases [2]. These diseases will result in the performance degradation of the main cable and seriously affect the long-term performance of the whole bridge.

A number of scholars have conducted relevant studies on the performance evaluation of main cables of large span suspension bridges. Matteo used the extremum distribution method to evaluate the safety factors of the main cables of Williamsburg Bridge [3]. On the other hand, Camo proposed a probabilistic method to evaluate the safety reliability of the main cable [4] Cremona proposed a probabilistic method to estimate the residual strength of the cable steel wire, which had been used to evaluate

\footnotetext{
${ }^{1}$ Shiqiao Xu, China Construction Eighth Engineering Division Corp., Ltd, Shanghai, China; E-mail: sqxu@tongji.edu.cn.
} 
the safety performance of the main cable of the Tangcaville Bridge [5]. Structural health monitoring (SHM) technology has been successfully applied to understand the loads, environment actions, and behaviors of a structure, thereby facilitating a new channel to evaluate the performance of main cables [6].

\section{Engineering Research Background}

The related study of the paper is conducted with reference to Xihoumen Bridge- the fourth longest suspension bridge in the world. The main cable of Xihoumen Bridge adopts prefabricated parallel wire strands and comprises 169 cable strands that run the length of the bridge from the north anchorage to the south anchorage. Each cable strand consists of 127 high-strength galvanized steel wires with a diameter of $5.25 \mathrm{~mm}$ and a nominal tensile strength of $1770 \mathrm{MPa}$. Figure 1 depicts the installation diagram of the anchor rope meter (figure $1 A$ ) and the detailed sensor location diagram (figure $1 B$ ) of the anchor rope meter (numbered CT1 to CT7) that is installed at the north anchorage of the left main cable. The data collected by CT3, which is installed at the bottom of the main cable with greater cable strand force, high humidity, from January 2015 to October 2020 and proneness to corrosion were selected to evaluate the long-term performance of the main cable in this paper.

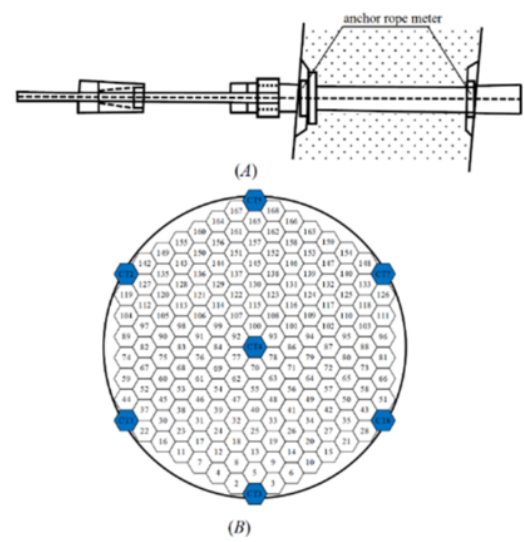

Figure 1. Anchor rope meter arrangement at the north anchorage of the left main cable of Xihoumen Bridge. (A) Installation diagram of anchor rope meter, (B) Detailed layout of anchor rope meter.

\section{Differential Stabilization of Time Series}

The long-term performance degradation of the main cable is mainly caused by the gradual corrosion and fracture of the main cable steel wire with the increase of service life. Moreover, because of the degradation of the structure itself, the monitoring data of the structure are not completely independent of each other at every time point. Therefore, it can be assumed that the data collected by each sensor in the bridge monitoring system comprise different time series. The analytical method of time series can be directly applied to analyze the monitoring data. 


\subsection{Difference Stationary of Time Series}

Stationarity is one of the most widely used time series properties whose statistical data characteristics do not change with time. It is difficult to establish analysis model through non-stationary time series. Therefore, the time series stationary processing method should be adopted for the main cable strand force monitoring data to eliminate the impact of long-term trend and periodic change on the time series. Figure $2 A$ shows the original time series of the main cable strand force data collected by CT3 anchor rope meter of Xihoumen Bridge from January 2015 to December 2018. The time series has annual cycle characteristics and has a gradual upward trend (figure $2 A$ ). Thus, the cable force data comprises a non-stationary time series. In order to eliminate the tendency and periodicity of the time series, according to the stationary process method specified in table 1 , the time series is processed by 365-step (one year) first-order difference. The time series after the first difference is shown in figure $2 \mathrm{~B}$. It is clear that the periodicity of the original main cable strand force time series is eliminated, but the upward trend still exists (figure $2 B$ ). Therefore, the time series after the difference is further differentiated to eliminate the upward trend. The data after twice difference is depicted in figure $2 C$. It can, therefore, be deduced that the time series of the main cable strand force after twice difference has no clear periodicity or tendency (figure $2 C$ ). Moreover, the main cable strand force value fluctuates around 0 and has a certain threshold (figure $2 C$ ), which indicates that the time series after twice difference does not have a clear non-stationary characteristic. Therefore, the stationarity test can be performed to determine whether the time series after twice differences is stationary.

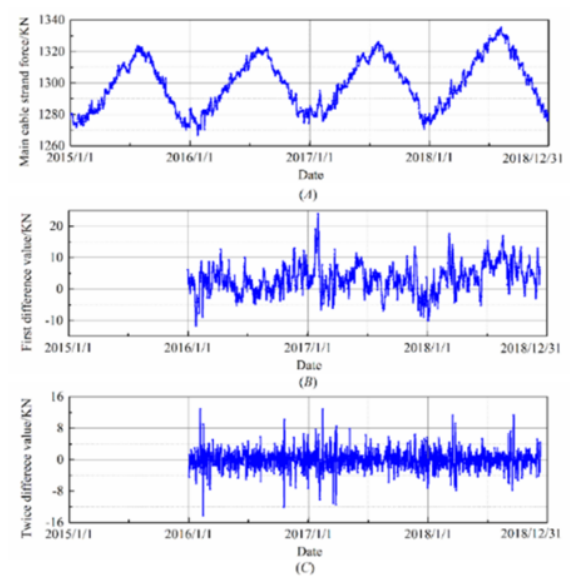

Figure 2. Original and post-differential time series of the main cable strand force of Xihoumen Bridge. (A) Original time series, (B) After 365-step first-order difference calculation, (C) After twice difference calculations.

\subsection{Stationarity Test of Time Series}

It is necessary to verify whether the original time series of the main cable strand force has been transformed from non-stationary to stationary before modeling time series. Graph test is the most popular method of verifying the stationarity of time series, which is simple, convenient, and can be utilized to identify model parameters [7]. Figure 3 depicts the sequence diagram of autocorrelation and partial autocorrelation coefficients 
of the original main cable strand force time series. The autocorrelation and partial autocorrelation function values of the original time series show the characteristic of 'Trailing', and the function value still far exceeds 0 at the 36th order lag. This indicates that the original time series is a non-stationary time series.

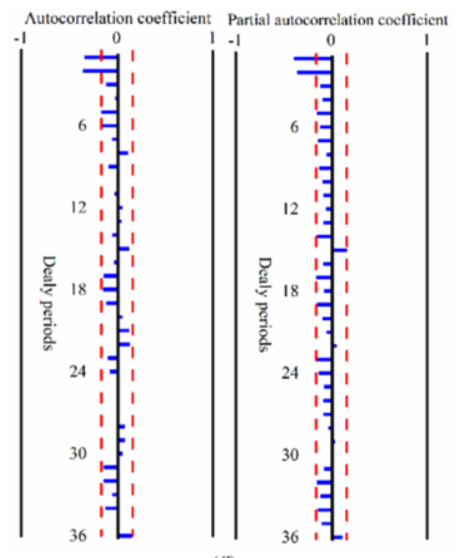

Figure 3. Stationarity test of main cable strand force time series. after stationary processing.

\section{Establishment of ARIMA Model}

Based on the original time series of main cable strand force of Xihoumen Bridge, a 365-step first-order difference and once more first difference are performed to eliminate the periodicity and tendency of the original time series. Moreover, the sequence diagram of the correlation coefficients of the time series after the twice-difference calculation is obtained. Therefore, it can be determined that $T=365$ and $d=2$ in the model $\operatorname{ARIMA}(p, d, q)_{T}$ that is established for the main cable strand force. Similarly, the autocorrelation and partial autocorrelation functions of the stationary time series are both 2-order delay truncations (figure 3)

Therefore, the ARIMA $(1,2,2)_{365}$ model was used to predict the main cable strand force of Xihoumen Bridge. The final analytic function of the model is as follows:

$$
(1+0.0051 L) \nabla^{2} Y_{365}=\left(1+1.9994 L-0.9939 L^{2}\right) \varepsilon_{t}
$$

Based on the $\operatorname{ARIMA}(1,2,2)_{365}$ model, the maximum annual main cable force values during the different operating years of Xihoumen Bridge can be predicted. The results of the prediction are shown in table 1.

Table 1. Maximum annual value of main cable strand force of Xihoumen Bridge.

\begin{tabular}{llllll}
\hline Operating years & 10 & 20 & 30 & 40 & 50 \\
\hline Main cable stand force $/ \mathrm{KN}$ & 1358.41 & 1371.56 & 1387.01 & 1405.45 & 1427.21 \\
\hline Operating years & 60 & 70 & 80 & 90 & 100 \\
\hline Main cable stand force $/ \mathrm{KN}$ & 1452.85 & 1482.23 & 1515.62 & 1553.28 & 1595.36 \\
\hline
\end{tabular}




\section{Discussion}

Our ultimate goal is to evaluate the long-term performance of the main cable. The main cable performance evaluation index during the operation period should not only consider the safety performance but also the durability and service performance. Therefore, the load degree index can be defined to evaluate the performance of the main cable in this paper, which is the ratio of actual cable force to the designed cable force. The calculation formula of load degree is shown in formula (2) as follows:

$$
D_{T}=\frac{\max \left(R_{T}\right)}{\sigma}
$$

where $D_{T}$ is the load degree of the main cable in the period of $T, R_{T}$ is the corresponding monitoring value of the main cable strand force in the period of $T$, and $\sigma$ is the design value of main cable. It should be considered that the factors that influence the main cable force do not only include vehicle load and wind load but also temperature load, which plays a key role. Therefore, $T$ is determined as $24 \mathrm{~h}$. Therefore, the daily average value of main cable strand force is selected to calculate the load degree index. Figure 4 shows the distribution and fitted curve of load degree index of Xihoumen Bridge in 2019.

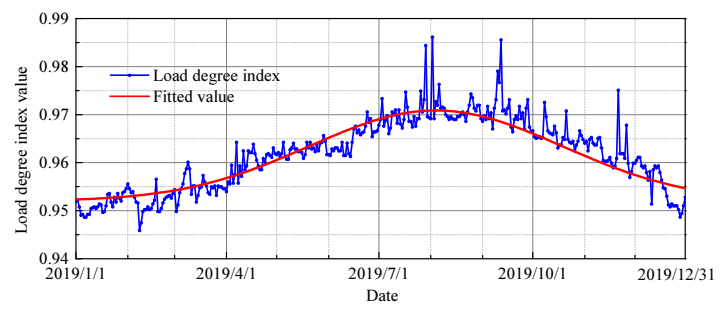

Figure 4. Distribution and fitted curve of load degree index of Xihoumen Bridge in 2019.

The variation characteristics can be deduced by fitting the distribution of load index. The load degree index increases gradually at first and then gradually decreases, which conforms to the trend of temperature change within a year (figure 4). Consequently, it proves that the load degree index is greatly affected by the temperature load. The load degree index mainly reflects the ratio of the actual main cable force to the designed cable force. The main cable performance grading standard of load degree index can be determined, as shown in table 2, through the analysis.

Table 2. Main cable performance grading standard of load degree index.

\begin{tabular}{lcccc}
\hline Performance level & I (Very good) & II (Good) & III (General) & IV (Poor) \\
\hline Load degree $\left(D_{T}\right)$ & $D_{T} \leq 1.05$ & $1.05<D_{T} \leq 1.12$ & $1.12<D_{T} \leq 1.23$ & $D_{T} \geq 1.23$ \\
\hline
\end{tabular}

It is, therefore, clear that the performance grading level of the main cable is mainly dominated by the maximum cable force of different operation times. Based on the definition of load degree index and the predicted value of main cable strand force (table 1), the long-term performance grading levels of the main cable in different operating periods were obtained and the detailed results are shown in table 3. 
Table 3. Main cable performance grading level of different operating periods.

\begin{tabular}{lcll|cl|cl|cl|cl|}
\hline Operating periods & 10 & & 20 & & 30 & & 40 & & 50 & \\
\hline \multirow{2}{*}{ Performance level } & $D_{T}$ & Level & $D_{T}$ & Level & $D_{T}$ & Level & $D_{T}$ & Level & $D_{T}$ & Level \\
& 0.99 & I & 1.01 & I & 1.01 & I & 1.02 & I & 1.04 & I \\
\hline Operating periods & 60 & & 70 & & 80 & & 90 & & 100 & \\
\hline \multirow{2}{*}{ Performance level } & $D_{T}$ & Level & $D_{T}$ & Level & $D_{T}$ & Level & $D_{T}$ & Level & $D_{T}$ & Level \\
& 1.06 & II & 1.08 & II & 1.10 & II & 1.13 & III & 1.16 & III \\
\hline
\end{tabular}

The value of load degree index of the main cable gradually increases with the increase of the operating periods (table 3). It indicates that the deviation between the actual value of the main cable strand force and the design value also increases gradually.appropriate maintenance methods and strategies (for example, main cable dehumidification system) should be adopted to ensure good performance of the main cable.

\section{Conclusions}

The long-term performance of the main cable has been evaluated in this paper from the perspective of the performance degradation problem of the main cable during the long-term operating periods of a suspension bridge. The main conclusions can be summarized as follows:

(1) The original time series of the main cable strand force of Xihoumen Bridge is a non-stationary time series. Thus, the periodicity of the original time series needs to be eliminated through a 365 -steps first difference calculation.

(2) The ARIMA model is established to predict the main cable force and the parameters of the model are identified by maximum likelihood estimation. The $\operatorname{ARIMA}(1,2,2)_{365}$ model is determined to predict the main cable strand force through model accuracy and validity verification. The calculation results show that the main cable strand force increases with the increase in the service life.

(3) By the definition of the load degree index, the long-term performance of the main cable has been discussed to be quantitatively evaluated. The performance level of the main cable gradually decreases with the increase of the operating periods. Therefore, appropriate maintenance and management measures must be adopted to ensure the long-term good performance of the main cable.

\section{Acknowledgements}

The study was jointly supported by the National Natural Science Foundation of China (Grant Nos. 51878493 and 51678437).

\section{References}

[1] Xu X, Huang Q, Ren Y, Zhao DY, Zhang DY, Sun HB. Condition evaluation of suspension bridges for maintenance repair and rehabilitation: a comprehensive framework. Structure and Infrastructure Engineering. 2019 Apr; 15(4): 555-567.

[2] Ma RJ, Xu SQ, Wang DL, Chen AR. Vehicle models for fatigue loading on steel box-girder bridges 
based on weigh-in-motion data. Structure and Infrastructure Engineering. 2018 Aug; 14(6): 701-713.

[3] Matteo J, Deodatis G, Billington. Safety analysis of suspension-bridge cables: Williamsburg bridge-closure. Journal of Structural Engineering. 1996 Jul; 122(7): 837-838.

[4] Camo S. Probabilistic strength estimates and reliability of damaged parallel wire cables. Journal of Bridge Engineering. 2003 Oct; 8(5): 297-311.

[5] Cremona C. Probabilistic approach for cable residual strength assessment. Engineering Structures. 2003 Feb; 25(3): 377-384.

[6] Li CX, Li Y, He J. Experimental study on torsional behavior of spatial main cable for a self-anchored suspension bridge. Advances in Structural Engineering. 2019 Oct; 22(14): 3086-3099.

[7] Ren Y, Xu X, Huang Q, Zhao DY, Yang J. Long-term condition evaluation for stay cable systems using dead load-induced cable Forces. Advances in Structural Engineering. 2019 May; 22(7): 1644-1656. 